\title{
Attitude to cervical cancer screening and sexual behaviour among HPV vaccinated young women-A qualitative pilot study*
}

\author{
Marie G. Oscarsson ${ }^{1,2 \#, ~ A n n a ~ Q v a r n s t r o ̈ m ~}{ }^{1}$, Tanja Tydén ${ }^{2}$ \\ ${ }^{1}$ School of Health and Caring Sciences, Linnaeus University, Kalmar, Sweden; ${ }^{*}$ Corresponding Author: marie.oscarsson@lnu.se \\ ${ }^{2}$ Department of Public Health and Caring Sciences, Uppsala University, Uppsala, Sweden \\ Received 23 April 2013; revised 26 May 2013; accepted 18 June 2013 \\ Copyright (C) 2013 Marie G. Oscarsson et al. This is an open access article distributed under the Creative Commons Attribution Li- \\ cense, which permits unrestricted use, distribution, and reproduction in any medium, provided the original work is properly cited.
}

\begin{abstract}
Objectives: To explore attitudes to cervical cancer screening and sexual behaviour among human papillomavirus (HPV)-vaccinated young women in southern Sweden. Methods: Sixteen women aged 17 - 26 years who had received the HPV vaccine were submitted to an individual semi-structured interview. Interviews were recorded and transcribed verbatim. The data were analysed qualitatively by means of latent content analysis. Results: The interviewees had a poor understanding of cervical cancer screening. After it was explained to them, they stated that they were willing to participate in the screening. The young women thought their future sexual behaviour would not be affected by the vaccination. They considered themselves to be more aware of it and less likely to engage in risky behaviour than their peers. They knew little about the relation among HPV, sexual transmission and cervical cancer, and they expressed a desire for more information. Conclusions: These young women who recently had been administered HPV vaccine had a limited understanding of the importance of participating in future cervical cancer screening. It is necessary that more and more appropriate information of cervical cancer screening be given on the occasion of the HPV vaccination.
\end{abstract}

Keywords: Cervical Cancer Screening; Human Papillomavirus; Sexual Behaviour; Vaccine; Young Women

\footnotetext{
*Declaration of interest: The authors report no conflicts of interest. The authors alone are responsible for the content and the writing of the paper.
}

\section{INTRODUCTION}

Human papillomavirus (HPV) is a sexually transmitted infection (STI). It is estimated that $50 \%-80 \%$ of sexually active women and men become infected at some point in their lives. Most of the 100 known HPV types cause asymptomatic infections, but some are implicated in the genesis of cervical cancer, anogenital cancer, oropharyngeal cancer and other neoplasias [1,2]. In Sweden, HPV prevalence was determined in women aged 15 - 23 years visiting youth clinics, and of 544 successfully analysed samples from non-vaccinated individuals, 62\% were positive for high-risk HPV types [2]. The best known is the association between HPV 16 and 18 and cervical cancer. Cervical cancer is one of the most preventable cancers due to its gradual development from the precursor lesions into invasive cancer, which usually takes ten to twelve years. Since this condition develops over a long period, abnormal changes can be detected and treated in early stages.

Each year approximately 450 of the 4.2 million women living in Sweden are diagnosed with cervical cancer, of whom 150 die from the disease [3]. This relatively low morbidity and mortality of cervical cancer has decreased by $50 \%$ and can be attributed to the extensive screening programme, conducted since the 1960's [4]. The counties administer the programs under the supervision of the National Board of Health and Welfare. The Swedish total population register is used for identification and receives information daily from the tax authori ties. The register includes information about births, deaths, migration, change of address, and change in civilstatus. Nowadays, Swedish women at 23 - 60 years of age are offered regular cervical cancer screening (CCS) at a low cost. The participation rate varies between different county councils and women in the youngest age group are less likely to participate than those who are 
older [5].

Today two different HPV vaccines are available. One protects against HPV strains 6, 11, 16 and 18, and the other one against 16 and 18 . The vaccine is most effective if given before the recipient's sexual debut and subsequent potential exposure to HPV [6]. In Sweden the quadrivalent vaccine has been included in the general child vaccination programme since the spring of 2012, and girls aged 10 - 12 are offered vaccination free of charge at school. County health services have also been encouraged to implement an expanded vaccination of girls aged 13 - 17 years—a so-called "catch-up". The quadrivalent HPV vaccine is available for women up to 45 of age but in Sweden women at 18 years of age and older must pay for the vaccination. The cost for the HPVvaccination at the time of the interviews was $3000 \mathrm{SEK} / €$ 325 for women and 1500 SEK/€ 162 for girls under 18. Pa- rental consent was required for the latter group. As the vaccine does not prevent all cervical carcinomas, it is essential that young women continue to submit to regular CCS. In Sweden, this is stated in the leaflets which should be given to the HPV-vaccinated women.

Prior to the introduction of the HPV vaccination, there were concerns, especially among parents, about the problems that could possibly ensue, for example, greater sexual risk taking behaviour [7-9], some young women may believe that they are vaccinated against STIs in general and, therefore, stop protecting themselves and no longer use condoms. Vaccinated women may also be lulled into a false sense of security leading to a reduced participation in CCS. This study is a part of a larger project with interview studies and public surveys which explore the understanding of HPV vaccination among parents [10], young women and men [11-13] and health professionals [14,15] in Sweden.

The purpose of this study was to assess the attitudes towards cervical cancer screening, and sexual behaviour, among HPV-vaccinated young women.

\section{METHODS}

The study population consisted of young women aged 17 - 26 years. The inclusion criteria required that participants could understand and speak Swedish, and were willing to talk to the researcher about vaccination against HPV. The young women were recruited through a purposeful sampling: that means selecting information-rich participants who can learn a great deal about the issues of central importance to the purpose. Recruitment was conducted through three vaccination clinics in mid-sized cities in Sweden. The nurse in charge at the clinic asked the young women, during vaccination visits, whether they would be willing to participate in the study. Those interested received written information about the aim and the procedure of the study. Those who were willing to participate provided informed consent. The recruitment of participants continued until no new information was obtained and redundancy achieved [16,17]. In total 17 vaccinated young women were asked to participate in the study. One woman declined to participate, the other 16 completed the interview. Background data of the participants are presented in Table 1.

The interviews with the young women were conducted, one to four weeks after the vaccination. Participants chose the location where the interviews would take place, which happened to be at work, at home and in public places. They were asked to complete a questionnaire providing background data. An interview guide with open questions was used. The questions analysed for this study were, "How do you think HPV vaccination may affect your future sexual behaviour?" and "How do you think HPV vaccination may affect your participation in CCS?” The researchers asked follow-up questions and repeated the answers when necessary. Each interview lasted between 30 - 60 minutes, and all were tape-recorded and transcribed verbatim. The study was approved by the Regional Ethics Committee for Human Research, Faculty of Health Sciences, Linköping University, Sweden (Dnr M214-08).

An inductive content analysis inspired by Graneheim and Lundman [18] was used to interpret the text. Three persons conducted the data analysis. According to the established procedures of content analysis, the transcribed interviews were read separately, after which content and meaning were discussed. Meaning units in the interviews matching the purpose of the study were

Table 1. Background characteristics of the 16 participants.

\begin{tabular}{cc}
\hline Mean age, years & 19.6 (range 17 - 26) \\
\hline Occupation & $\mathrm{n}=15$ \\
Student & $\mathrm{n}=1$ \\
Employee & \\
Marital status & $\mathrm{n}=15$ \\
Single & $\mathrm{n}=1$ \\
Cohabitant & \\
Sexually debuted & $\mathrm{n}=11$ \\
Yes & $\mathrm{n}=5$ \\
No & $\mathrm{n}=4$ \\
\hline Mean age at sexual debut, years & $\mathrm{n}=5$ \\
\hline Number of lifetime sexual partners & \\
\hline None & \\
\hline Nang 17 - 26) & \\
\hline
\end{tabular}


colour-marked and meaning-bearing units were condensed, abstracted and given a code based on what they represented. Out of the similarities and differences in the condensed material subcategories and categories took form. Besides condensing the text, the interviews were also read in their entirety so as to see the full text in context. The final stage in the analysis is to bring the data together, by selecting various examples from the text, which links the categories and the quotations together. When uncertainty arose on how sentences should be interpreted, the material was discussed among the authors until consensus emerged [18].

\section{RESULTS}

\subsection{Low Awareness of Cervical Cancer Screening}

The understanding of CCS for seven of the young women participating in the study was so poor that they could not answer questions about how or if they thought the vaccine would affect future participation in CCS. Although most interviewees had heard about CCS they had various understandings of what it meant.

"I have only heard about it, but what it means, I cannot say."

"That's what you do from the age of 21 years, and they check if there are any lesions."

When the interviewer explained what CCS was, all young women stated that they did not believe that the vaccination would affect their future participation. In general they had a positive attitude toward medical controls and examinations.

"It is something I definitely intend to do if I be invited, it's the same thing with mammography and all those things, if there are resources, I think you should make use of it"

Few young women had a relevant understanding of the level of protection against cervical cancer that the vaccine could provide. They mentioned the importance of continuing to attend CCS and expressed a feeling of fear of what might happen if they refrained.

"... of course, we had a long chat about how it really doesn't protect against everything, that it's only a certain amount you protect yourself against with this."

Only one woman mentioned that she had received information about CCS in connection to the vaccination.

"I think, he mentioned that it is important to continue to participate in CCS ... he did not emphasise it."

Although they stated that they would participate in CCS, they had some doubts about how other young women would behave. It was perceived that their participation in CCS could diminish if they believed the vaccine gave full protection and that further checks were unnecessary.
"But I think for sure that there's lots of girls who think that now they're vaccinated, it's all over with ... but personally, I'll probably go and do it anyway [attend CCS] ... I think ..."

One young woman thought that medical examinations that cost money could be seen as less important and might have a negative effect on participation.

“People aren't as motivated. I mean, OK, it doesn't really cost anything, but by having to pay then I think that people might not do it because it doesn't seem as important, and eh... You might get lazier and not bother as much if you're vaccinated, like you know you're vaccinated and that's enough.”

\subsection{Will Protect Myself}

The young women did not expect future sexual behaviour to be affected by the vaccination. They saw no reason why they would be less cautious since there are other STIs that make protection necessary.

"Well, no ... I feel the same now as I did before about having to protect myself. I mean, it's not protection against everything else out there."

But one woman's understanding was flawed.

“... I was really happy of course because if in the future I get involved with someone sexually then I'm also more protected against STIs, ... A lot of women get chlamydia, so I'm really glad, I mean, that I'm vaccinated.”

The young women did not think they had been given enough information regarding HPV and the vaccine, at school or by health services, and wished they had received more. They had a vague understanding about the relation between HPV and sexual transmission and the vaccine.

“... in my high school we didn't have sex education so we weren't aware of the disease or the vaccine. Since then, I've been to the vaccination clinic and seen some flyers but not much more than that."

Vaccination and discussions concerning the decision to vaccinate led in some cases to increased awareness of sexual health. The young women felt that their knowledge and interest in HPV, other STIs, and safe sex had improved in connection to the HPV vaccination, and they had become aware of the subject in the media, at school, via health services and friends and family.

“... I've really thought a lot about protecting myself much more now with condoms and that kind of thing. So actually, it has, you know, em, changed. Changed my opinion about it, so to speak. Otherwise, I never thought about it before, at all."

Participants thought their own future sexual behaviour differed from that of other young women. They described a feeling of being protected and a sense of security after the vaccination, and perceived themselves as 
more knowledgeable and less likely to engage in risk behaviour than their peers. They believed that other young women who already had a risk behaviour would continue this way regardless of whether they were vaccinated or not.

"But I've always been careful about protecting myself and all that, but ... there might still be a disadvantage, that people start thinking, "I don't need to go to checkups, I'm vaccinated." ... I think that those who protect themselves do it regardless, and those who don't, don't."

One of the interviewees did not protect herself against STIs, neither before nor after the vaccination; she did not consider catching a STI was particularly serious and was not careful with protection for that reason. She was more concerned with not getting pregnant in new sexual relationships.

"I probably think about it all wrong, I'm on the pill and always only think of not getting pregnant."

\section{DISCUSSION}

In spite of their having been recently vaccinated against HPV these young women had only a limited knowledge about the relation between HPV, sexual transmission and cervical cancer screening. When this was explained they stated they had a positive attitude towards participation in CCS. The young women thought future sexual behaviour would not be affected by the vaccination. They considered themselves to be more knowledgeable and less likely to engage in risk behaveiour than their peers. They expressed a desire for more information.

It is surprising that so many young women in our study had such a limited understanding of CCS. The Swedish National Board of Health and Welfare has emphasised the importance of providing information about CCS at the time of vaccination and it is clearly stated in the leaflets provided to women on the occasion of HPV vaccination. If this information is not received or understood young women may be lulled into a false sense of security that the vaccination makes future CCS superfluous, which is reported by parents in a study from UK [19]. This could result in a lower attendance of CCS and thereby weaken the effectiveness of existing programmes. For this reason, it is essential to continue to inform and to motivate women to attend CCS, including those who are HPV vaccinated.

One unanswered question is whether the young women in our study had received information but failed to absorb it or whether information was never given. In case the young women had failed to absorb the written information perhaps an alternative method of providing information is necessary. We suggest that both verbal and written information be provided at the time of vaccination. Additionally, if women are invited to CCS pro- grammes by letters it should be made clear in the latter that participation in the CCS is necessary even for HPVvaccinated women. A final suggestion is that healthcare professionals at youth centres motivate young women by talking about CCS since it is the women in the youngest age groups who participate the least in CCS in Sweden [5].

The young women in our study had a positive attitude towards safe sexual behaviour. This result is in line with two recently published studies [20,21]. Mather et al. [21] found that vaccinated women were more inclined to practising safer sex than unvaccinated ones and similar observations were made by Liddon et al. [20] who reported that vaccinated women used condoms more frequently than those who were not. One explanation for these findings could be that the HPV vaccinated woman is more motivated to have a safer sexual health. If this is the case, unvaccinated women are in greater need information such condom uses and encouraging CCS participation than the vaccinated women. Parents have also raised concerns about the vaccine potentially increasing risky sexual behaviour [7-9]. Similar worries have been expressed about condoms and emergency contraceptive pills (ECPs) but studies have shown the opposite: initiatives providing adolescents free access to condoms [22] or ECPs [23] did not increase sexual risk taking. A majority of the young women in the study had had their sexual debut which might have an impact on their attitude to safe sexual behaviour.

The finding that young women consider themselves as being more responsible and less likely to engage in risky behaviour than their peers has also been reported by Marlow and co-authors [24]. Statements about others are less personal and may, therefore, make it easier for interviewees to respond more truthfully, but more work is needed to see which predictor of behaviour is the most reliable.

Many of the young women in our study, as in others, had limited knowledge about HPV, and did not understand the relation between HPV, sexual transmission and cervical cancer. HPV vaccination is marketed today primarily as a means of preventing cervical cancer. Advertising of the HPV vaccine should also focus on that the vaccine does not give full protection. Therefore, after vaccination, one should keep using condoms and participating in CCS programmes. The young women in our study expressed a desire for more information from school and healthcare professionals, which was also brought to light by a previous study [13]. However, several surveys $[14,25,26]$ have shown that healthcare professionals do not feel sufficiently knowledgable about HPV to inform young people. It is important that teachers and healthcare professionals are given suitable resources so that they feel confident in their role talking to 
young people about HPV.

\section{Strengths and Weaknesses of the Study}

Our intention was not to report data which could be generalised to the population at large. Qualitative research is particularly concerned with the way people understand and give meaning to the social world, and the aim is not to find an objective truth $[16,17]$. Another limitation of this study is the small sample of participants; the sample was restricted to young women who had chosen to be HPV vaccinated. They may have had a greater interest in HPV and health. However, the low awareness of CCS among these young women could indicate an even lower awareness among a broader public.

\section{CONCLUSIONS AND IMPLICATIONS}

It will take several years before the first cohort of HPV-vaccinated young women be invited to submit to CCS, and as many to evaluate the impact of HPV vaccination on sexual behaviour and attendance of CCS programmes. Our findings give an insight only into young women's reasoning on the subject. Our study highlights the limited understanding HPV-vaccinated young women have of the importance of participating in future CCS.

This research provides a basis for further research on sexual behaviour and participation in CCS programmes. It is needed to investigate young women's lack of awareness of the need for cervical smears after HPV vaccination, and to plan interventions which promote their attendance of CCS.

\section{ACKNOWLEDGEMENTS}

We would like to thank the young women who kindly agreed to participate in the study. Special thanks also to Anna-Karin Hannerfors, who conducted some of the interviews; Marie Mirus, who participated in interpreting and analysing the interviews; and Simon Beck for revising the English. The study was funded by the Medical Research Council of Southeast Sweden.

\section{REFERENCES}

[1] Zur Hausen, H. (2006) Papillomavirus infections: A major cause of human cancer. Infections causing human cancer. Wiley-VCH Verlag, Weinheim. doi:10.1002/3527609318

[2] Ramqvist, T., Du, J., Lunden, M., Ahrlund-Richter, S., Ferreira, J., Marions, L., Sparen, P., Andersson, S. and Dalianis, T. (2011) Pre-vaccination prevalence of human papillomavirus types in the genital tract of 15-23-year-old women attending a youth health clinic in Stockholm, Sweden. Scandinavian Journal of Infectious Diseases, 43, 115-121. doi:10.3109/00365548.2010.526957
[3] Sveriges Officiella Statistik (2009) Causes of death 2007. Socialstyrelsen, Stockholm.

[4] Bergstrom, R., Sparen, P. and Adami, H.O. (1999) Trends in cancer of the cervix uteri in Sweden following cytological screening. British Journal of Cancer, 81, 159-166. doi:10.1038/sj.bjc.6690666

[5] Rodvall, Y., Kemetli, L., Tishelman, C. and Tornberg, S. (2005) Factors related to participation in a cervical cancer screening programme in urban Sweden. European Journal of Cancer Prevention, 14, 459-466. doi:10.1097/01.cej.0000178078.21910.da

[6] Schiller, J.T., Castellsague, X., Villa, L.L. and Hildesheim, A. (2008) An update of prophylactic human papillomavirus L1 virus-like particle vaccine clinical trial results. Vaccine, 26, K53-K61. doi:10.1016/j.vaccine.2008.06.002

[7] Marlow, L.A., Waller, J. and Wardle, J. (2007) Parental attitudes to pre-pubertal HPV vaccination. Vaccine, 25, 1945-1952. doi:10.1016/j.vaccine.2007.01.059

[8] Brewer, N.T. and Fazekas, K.I. (2007) Predictors of HPV vaccine acceptability: A theory-informed, systematic review. Preventive Medicine, 45, 107-114. doi:10.1016/j.ypmed.2007.05.013

[9] Bernat, D.H., Harpin, S.B., Eisenberg, M.E., Bearinger, L.H. and Resnick, M.D. (2009) Parental support for the human papillomavirus vaccine. Journal of Adolescent Health, 45, 525-527. doi:10.1016/j.jadohealth.2009.04.014

[10] Grandahl, M., Tyden, T., Gottvall, M., Westerling, R. and Oscarsson, M. (2012) Immigrant women's experiences and views on the prevention of cervical cancer: A qualitative study. Health Expectations. (Epub ahead of print) doi:10.1111/hex.12034

[11] Gottvall, M., Tyden, T., Hoglund, A.T. and Larsson, M. (2010) Knowledge of human papillomavirus among high school students can be increased by an educational intervention. International Journal of STD \& AIDS, 21, 558562. doi:10.1258/ijsa.2010.010063

[12] Oscarsson, M.G., Hannerfors, A.K. and Tyden, T. (2012) Young women's decision-making process for HPV vaccination. Sexual \& Reproductive Healthcare, 3, 141-146. doi:10.1016/j.srhc.2012.10.002

[13] Gottvall, M., Larsson, M., Hoglund, A.T. and Tyden, T. (2009) High HPV vaccine acceptance despite low awareness among Swedish upper secondary school students. The European Journal of Contraception and Reproductive Health Care, 14, 399-405. doi:10.3109/13625180903229605

[14] Oscarsson, M.G., Dahlberg, A. and Tyden, T. (2011) Midwives at youth clinics attitude to HPV vaccination and their role in cervical cancer prevention. Sexual \& Reproductive Healthcare, 2, 137-142. doi:10.1016/j.srhc.2011.09.001

[15] Gottvall, M., Tyden, T., Larsson, M., Stenhammar, C. and Hoglund, A.T. (2011) Challenges and opportunities of a new HPV immunization program perceptions among Swedish school nurses. Vaccine, 29, 4576-4583. doi:10.1016/j.vaccine.2011.04.054 
[16] Creswell, J.W. (2007) Qualitative inquiry \& research design: Choosing among five approaches. 2nd Edition, SAGE, Thousand Oaks.

[17] Polit, D.F. and Beck, C.T. (2004) Nursing research: Principles and methods. 7th Edition, Lippincott Williams \& Wilkins, Philadelphia.

[18] Graneheim, U.H. and Lundman, B. (2004) Qualitative content analysis in nursing research: Concepts, procedures and measures to achieve trustworthiness. Nurse Education Today, 24, 105-112. doi:10.1016/j.nedt.2003.10.001

[19] Henderson, L., Clements, A., Damery, S., Wilkinson, C., Austoker, J. and Wilson, S. (2011) “A false sense of security”? Understanding the role of the HPV vaccine on future cervical screening behaviour: A qualitative study of UK parents and girls of vaccination age. Journal of Medical Screening, 18, 41-45. doi:10.1258/jms.2011.010148

[20] Liddon, N.C., Leichliter, J.S. and Markowitz, L.E. (2012) Human papillomavirus vaccine and sexual behavior among adolescent and young women. American Journal of Preventive Medicine, 42, 44-52. doi:10.1016/j.amepre.2011.09.024

[21] Mather, T., McCaffery, K. and Juraskova, I. (2012) Does HPV vaccination affect women's attitudes to cervical cancer screening and safe sexual behaviour? Vaccine, 30,
3196-3201. doi:10.1016/j.vaccine.2012.02.081

[22] Kirby, D. (2002) The impact of schools and school programs upon adolescent sexual behavior. Journal of Sex Research, 39, 27-33. doi:10.1080/00224490209552116

[23] Ekstrand, M., Larsson, M., Darj, E. and Tyden, T. (2008) Advance provision of emergency contraceptive pills reduces treatment delay: A randomised controlled trial among Swedish teenage girls. Acta Obstetricia et Gynecologica Scandinavica, 87, 354-359. doi:10.1080/00016340801936024

[24] Marlow, L.A., Forster, A.S., Wardle, J. and Waller, J. (2009) Mothers' and adolescents' beliefs about risk compensation following HPV vaccination. Journal of Adolescent Health, 44, 446-451. doi:10.1016/j.jadohealth.2008.09.011

[25] Brotherton, J.M., Leask, J., Jackson, C., McCaffery, K. and Trevena, L.J. (2010) National survey of general practitioners' experience of delivering the National Human Papillomavirus Vaccination Program. Sexual Health, 7, 291-298. doi:10.1071/SH09135

[26] Tafuri, S., Martinelli, D., Vece, M.M., Quarto, M., Germinario, C. and Prato, R. (2010) Communication skills in HPV prevention: An audit among Italian healthcare workers. Vaccine, 28, 5609-5613. doi:10.1016/j.vaccine.2010.06.028 